\title{
Les quasicristaux : une recherche pluridisciplinaire exemplaire
}

Denis Gratias (denis.gratias@onera.fr)

Laboratoire d'étude des microstructures (LEM, CNRS-ONERA), BP 72, 29 avenue de la Division Leclerc, 92322 Chatillon Cedex

Le prix Nobel de chimie 2011 a été décerné à Daniel Shechtman pour sa découverte en 1982 des quasicristaux.

Nous proposons ici de situer ce travail dans le contexte de l'époque, et de montrer quelques points remarquables qui illustrent en quoi cette découverte a significativement élargi notre conception de l'ordre spatial dans les solides. On verra en particulier que la recherche sur le sujet est fondamentalement pluridisciplinaire, impliquant à la fois les mathématiciens, les physiciens et les chimistes. À cet égard, le CNRS et le CEA de l'époque, suivis de la DFG allemande, ont été remarquablement réactifs et efficaces pour fédérer les laboratoires issus de ces diverses disciplines et faire ainsi de l'Europe un leader mondial dans ce domaine.

Les termes suivis d'un astérisque $\left(^{*}\right)$ sont explicités dans le glossaire, p. 16.
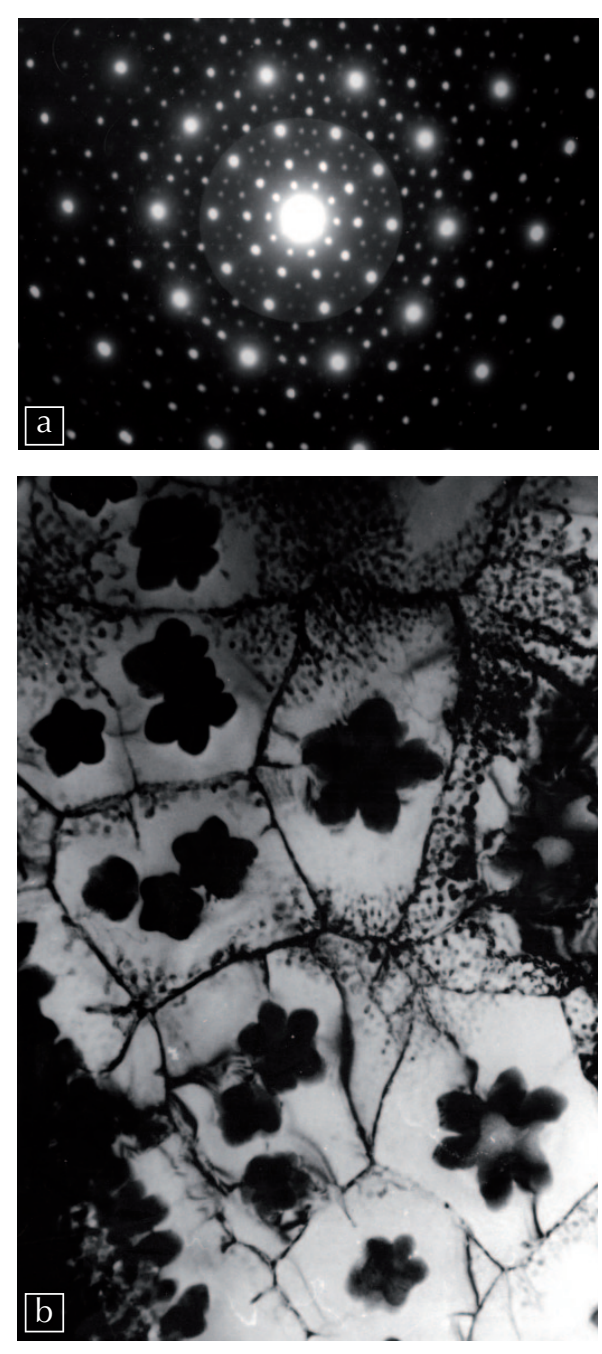

1. Diagramme de diffraction électronique (a) selon une orientation de symétrie 5 , et image de microscopie électronique à transmission (b) des précipités dendritiques de phase icosaédrique en forme de fleurs noires, poussant au sein de grains d'aluminium dans un alliage $\mathrm{Al}_{6} \mathrm{Mn}$ rapidement solidifié. L'image (b) montre la morphologie de l'alliage. La taille moyenne des précipités est de quelques microns.

\section{Le contexte de la découverte}

C'est le 8 avril 1982 que Dan Shechtman, enseignant-chercheur israélien du Technion (Haifa, Israël), observe au microscope électronique à transmission la première figure de diffraction de symétrie 5 (" quinaire ") qui sera la signature emblématique des quasicristaux (fig. 1a). Il est alors chercheur invité au National Bureau of Standards (NBS, Gaithersburg, USA) - aujourd'hui le National Institute of Standards and Technology (NIST) - et travaille sur la métallurgie hors d'équilibre des alliages à base d'aluminium faiblement alliés obtenus par trempe rapide. C'est en observant la microstructure de l'alliage $\mathrm{Al}_{6} \mathrm{Mn}$ qu'il voit une remarquable précipitation dendritique (fig. 1b) qui diffracte les électrons très fortement selon certaines orientations privilégiées, comme dans les cristaux, mais avec une symétrie globale icosaédrique* (fig. 1a) incompatible avec la symétrie de translation cristalline ${ }^{(1)}$ !

Les analyses usuelles pour débusquer les éventuelles macles - petits cristaux de même nature orientés différemment les uns par rapport aux autres et superposés, qui seraient responsables de cette symétrie quinaire globale - se révèlent négatives. D. Shechtman comprend qu'il a affaire à une organisation ordonnée originale de l'état solide, qui ne semble rentrer dans aucun schéma de la cristallographie.

Et pourtant en 1972, le mathématicien français Yves Meyer (Prix Gauss 2010) publie un ouvrage sur l'analyse harmonique, qui reprend certains de ses résultats plus anciens sur les nombres algébriques [1]. Il invente ainsi des ensembles non périodiques, dits " harmonieux ", et dont le support de la transformée de Fourier est un ensemble dénombrable de points ! 


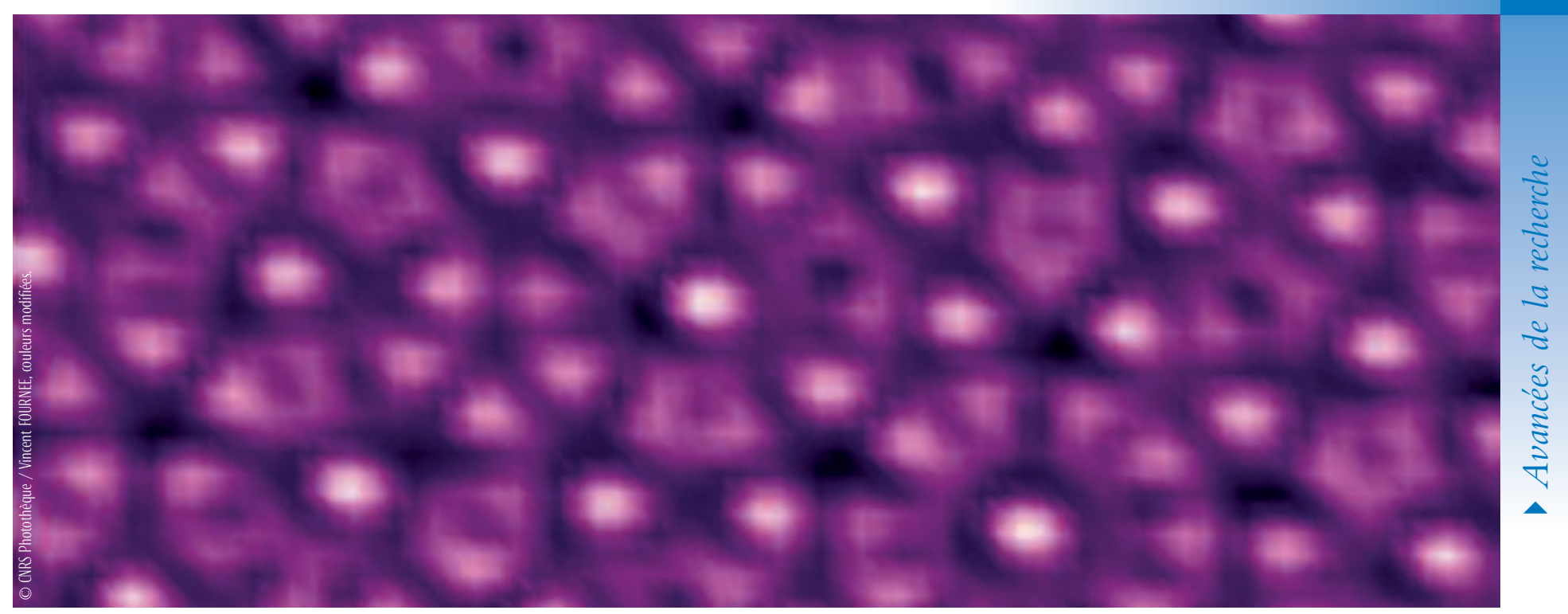

Image par microscopie à effet tunnel d'une surface de symétrie d'ordre 5 d'une phase quasi-cristalline AIPdMn de 73 x 29 nm². (Source : Institut Jean Lamour, Nancy.)

En 1974, le physicien théoricien anglais Roger Penrose trouve un ensemble de deux types de tuiles munies de règles d'accolement telles que les pavages résultants sont ordonnés apériodiques et de symétrie moyenne quinaire (voir image de couverture) [2]. Martin Gardner vulgarise ces pavages en leur consacrant un article célèbre dans Scientific American en 1977

En 1981, le cristallographe anglais Alan L. Mackay s'en inspire pour imaginer des assemblages atomiques dans un article visionnaire [3], "De Nive Quiquangula: on the pentagonal snowflake". Parmi ses conclusions, celle-ci : "it gives an example of a pattern of the type which might well be encountered but which might go unrecognized if unexpected...”. Finalement, en 1982, il montre la première diffraction optique par laser d'un pavage de Penrose [3] : une collection de spots fins se répartissant en couronnes de symétrie décagonale !

Ce n'est qu'au printemps 1984, soit deux ans après la découverte initiale, qu'Ilan Blech, professeur au Technion, convaincu par les résultats expérimentaux de D. Shechtman, construit un premier modèle structural en agrégeant au hasard des icosaèdres de même orientation et connectés deux à deux par une arête. Il en résulte une structure qui, quoique de nature aléatoire, possède d'exceptionnelles propriétés de diffraction : calculée par simulation numérique, l'intensité diffractée se localise sur un ensemble (apparemment) discret de vecteurs d'ondes correspondant à ceux observés par Shechtman. Malgré sa très faible densité atomique, ce modèle est capital : il est le premier de nouveaux édifices appelés les pavages aléatoires ("random tilings"), et qui vont rapidement devenir essentiels dans le cadre de la quasicristallographie.
D. Shechtman et I. Blech rassemblent leurs travaux et écrivent alors un article [4] soumis au Journal of Applied Physics. Celui-ci, refusé au motif qu'il est d'un intérêt limité pour les physiciens, est finalement envoyé le 2 octobre 1984 à Metallurgical Transactions, qui le publiera courant 1985. Entretemps, D. Shechtman convainc John Cahn, senior scientist au NBS, de l'existence expérimentale d'une symétrie quinaire intrinsèque possible dans un solide ordonné. Cahn propose alors d'écrire un article très concis et factuel pour la Physical Review Letters (PRL), autour de la question fondamentale d'une possible diffraction ponctuée selon une symétrie icosaédrique pour une assemblée non périodique d'atomes. Il s'assure la collaboration de Denis Gratias, rapidement convaincu à son tour, sur l'aspect mathématique du spectre. Celui-ci exhume les travaux de Bohr et Besicovic sur la presque périodicité, et confirme qu'aucune règle mathématique ne s'oppose à l'existence possible d'une diffraction de Bragg non périodique. Encore que ne proposant pas de modèle spécifique, l'article [5], soumis le 9 octobre 1984, est immédiatement accepté par PRL et publié le 12 novembre 1984, bien avant celui de Met. Trans., dont il est pourtant le successeur d'une semaine.

\section{Les premières recherches}

Les choses vont alors très vite. Dov Levine et Paul Steinhardt publient le 24 décembre 1984 un article [6] annonçant la possible existence d'un nouveau type de solides, les cristaux quasipériodiques, qu'ils nomment " quasicristaux ", et qui possèdent un spectre de diffraction dense dénombrable. Leurs résultats théoriques correspondent parfaitement aux diagrammes expérimentaux de
Shechtman : les précipités de l'alliage $\mathrm{Al}_{6} \mathrm{Mn}$ rapidement solidifié sont sans aucun doute les premiers représentants de ces nouveaux " quasicristaux ". Ainsi, si la découverte des quasicristaux date du printemps 1982, la recherche sur le sujet ne démarre vraiment que fin 1984.

En janvier 1985, à l'Institut des Hautes Études Scientifiques à Paris, a lieu un atelier de cristallographie mathématique organisé par Marjorie Senechal et Louis Michel. Shechtman, Cahn et Gratias y sont conviés, et ce dernier présente les quasicristaux dans le contexte de la presque périodicité en posant de nombreuses questions. Michel Duneau et André Katz [7] sont dans l'assistance et répondent point par point à toutes les questions en s'appuyant sur une méthode de coupe et projection (voir encadré 1, p. 15), lumineuse de simplicité - et découverte indépendamment par l'Américain V. Elser [8] et les Russes P. Kalugin, A. Kitayev et L. Levitov [9] -, qu'ils ont mise au point pour engendrer les pavages de Penrose. Ce jour-là, le cadre géométrique des quasicristaux est publiquement explicité et entre, en la généralisant, dans la description des phases incommensurables proposée par le cristallographe hollandais P.M. de Wolff [10] et formalisée par A. Janner et T. Janssen [11].

Une collaboration de près de vingt ans s'en suivra entre les deux groupes. Louis Michel, enthousiaste, décide d'organiser avec Gratias un atelier à l'École des Houches, qui aura lieu en mars 1986 grâce au concours des départements SPM, Chimie et PIRMAT du CNRS, l'Institut de recherche fondamentale du CEA, Péchiney, la société Elf-Aquitaine et IBM France. 
$>>>$

De nombreuses personnalités françaises témoignent de leur intérêt pour le sujet, parmi lesquelles André Guinier, Michel Fayard, Jacques Friedel et Georges Martin. Et si Shechtman dit avoir rencontré des difficultés à convaincre les scientifiques, sa découverte a été immédiatement accueillie en France dans la plus jubilatoire effervescence scientifique : aucune "révolution" n'aura été plus aisée. Tel n'est pas le cas à l'époque aux USA. Le célèbre chimiste cristallographe Linus Pauling s'insurge dans une lettre [12] à Nature du 10 octobre 1985 contre ces nouveaux objets qu'il attribue à un maclage multiple de cristaux cubiques, nonobstant les clichés de microscopie électronique haute résolution (fig. 2) de Richard Portier et al., publiés en mai. Il conclut en effet "crystallographers can now cease to worry that the validity of one of the accepted bases of their science has been questioned". Cette prise de position brutale est immédiatement réfutée par une série de lettres [13] à Nature du 9 janvier 1986, "Pauling's model not universally accepted”, signées par plusieurs groupes, Cahn, Gratias et Shechtman, le cristallographe britannique Alan Mackay, les physiciens américains P.A. Bancel, P. Stephens et A. Goldman, et le canadien A.A. Berezin, mais aucun cristallographe américain..

L'opposition tenace de Linus Pauling aux concepts quasicristallins va influer sur la dynamique de recherche des différents pays sur le sujet. Faute de cristallographes, les physiciens américains vont se pencher plutôt vers les modèles de pavages aléatoires et s'intéresser aux aspects entropiques et thermodynamiques de ces solides alors que les Européens, indifférents aux élucubrations de Linus Pauling, vont développer plutôt les aspects structuraux, phases icosaédriques en France et décagonales en Allemagne, en s'intéressant parallèlement aux propriétés physiques.

\section{Les structures atomiques des quasicristaux}

Très vite, de nouvelles structures quasicristallines vont être découvertes : la phase décagonale, identifiée par L. Bendersky au NIST [14], puis une série de phases icosaédriques dans des alliages ternaires à base d'aluminium (AlMnSi). Mais les recherches structurales vont prendre un véritable essor à partir de juillet 1986, quand Pierre Sainfort et Bruno Dubost, chercheurs au Centre de recherche Cegedur-Péchiney,
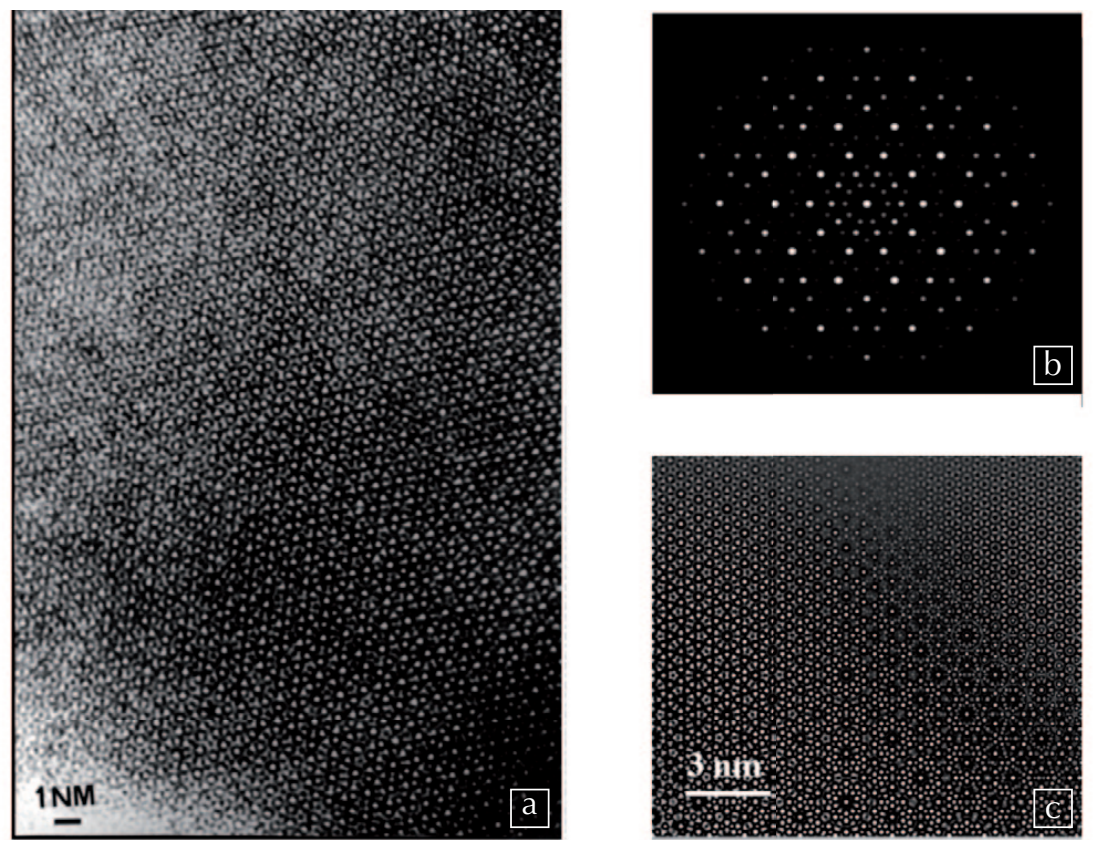

2. (a) Cliché de microscopie électronique haute résolution d'un alliage $\mathrm{Al}_{6} \mathrm{Mn}$ rapidement solidifié, effectué en janvier 1985 par R. Portier au CECM-Vitry. La distribution d'intensité de l'image est directement corrélée à celle de la densité électronique locale, projetée selon l'axe d'observation. La symétrie locale icosaédrique est présente jusqu'à l'échelle atomique, excluant ainsi le maclage multiple comme explication possible de la diffraction. (b) Résultat d'une simulation numérique de diffraction électronique dynamique du même alliage en fonction de la défocalisation et de l'épaisseur de l'échantillon. (c) Reconstruction de la structure atomique de cet alliage à partir de l'image de diffraction (b). Noter une bonne adéquation avec l'image expérimentale (a). On distingue deux types au moins d'amas atomiques imbriqués les uns dans les autres de façon parfaitement ordonnée.

annoncent la découverte [15] du premier quasicristal stable de taille macroscopique, obtenu par solidification lente d'un alliage $\mathrm{Al}-\mathrm{Li}-\mathrm{Cu}(\mathrm{Mg})$.

Les premières déterminations structurales par diffraction de rayons $\mathrm{X}$ et de neutrons sur monoquasicristaux vont alors se multiplier, avec l'utilisation explicite de la méthode de coupe : un objet quasi-périodique de dimension $d$ est une coupe $d$-dimensionnelle d'un objet périodique de dimension $\mathrm{N}>d$ (voir encadré 1).

Le chercheur japonais A. Tsai [16] va accélérer le processus expérimental de détermination structurale en découvrant coup sur coup les phases icosaédriques stables dans les systèmes ( $\mathrm{Al}, \mathrm{Cu}, \mathrm{Fe})$ et (Al, Pd, Mn). En novembre 1990, M. Cornier-Quiquandon et al. [17] réalisent par diffraction de neutrons au Laboratoire Léon Brillouin la première détermination structurale sur monoquasicristal de l'alliage icosaédrique $\mathrm{Al}_{62} \mathrm{Cu}_{25.5} \mathrm{Fe}_{12.5}$; ils étaient guidés par les travaux de Pierre Guyot et Marc Audier [18], qui proposèrent les premières unités structurales de symétrie locale icosaédrique (plus tard désignées par amas de Mackay) à partir des motifs des phases cristallines $\alpha$-(AlFeSi) et $\alpha$-(AlMnSi) (fig. 3). Le résultat est surprenant de simplicité : la structure est descriptible à l'aide de trois surfaces atomiques seulement, localisées aux sites de haute symétrie du réseau à six dimensions. Un an plus tard, en 1992, M. Boudard et al. [19] obtiennent un résultat analogue avec l'alliage $\mathrm{Al}_{70.3} \mathrm{Pd}_{21.4} \mathrm{Mn}_{8.3}$ par diffraction des rayons $\mathrm{X}$ à l'ESRF.

La solidité de la description cristallographique à $\mathrm{N}$-dimensions est aujourd'hui bien reconnue et utilisée dans le monde entier ; plusieurs centaines de phases quasicristallines, essentiellement icosaédriques et décagonales, ont été identifiées, dont les plus récentes dans les systèmes ( $\mathrm{Zn}, \mathrm{Mg}, \mathrm{Ho})$, ( $\mathrm{Zn}, \mathrm{Sc}, \mathrm{Mn})$ et $(\mathrm{Cd}, \mathrm{Yb})$.

\section{Quelques propriétés physiques}

Les phases icosaédriques ont été très tôt reconnues [20] comme des phases de HumeRothery ${ }^{\star}$, avec présence d'un pseudo-gap ${ }^{\star}$ au voisinage de l'énergie de Fermi. A. Tsai s'est systématiquement fié au critère phénoménologique d'analyse de stabilité en terme du nombre d'électrons par atome e/a pour trouver de nouveaux quasicristaux stables. 


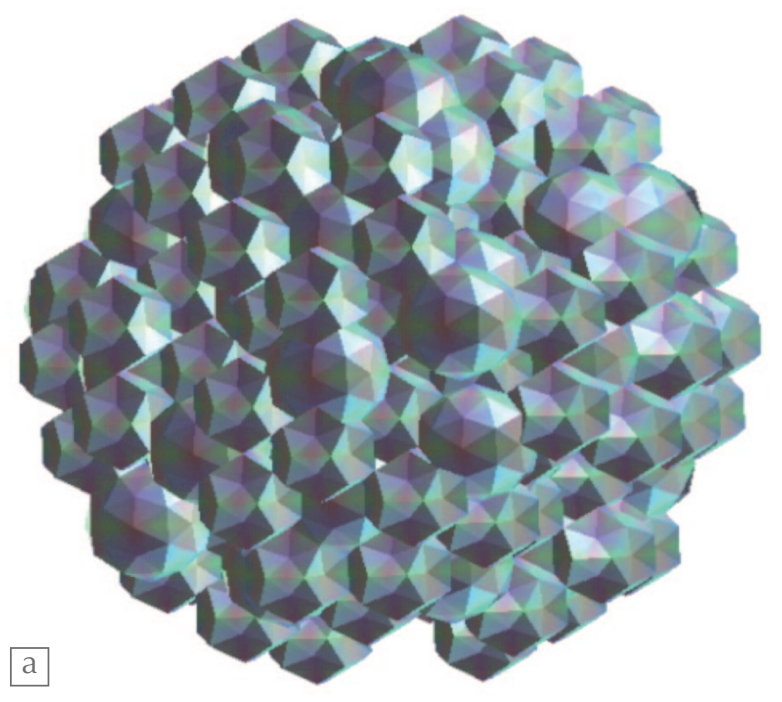

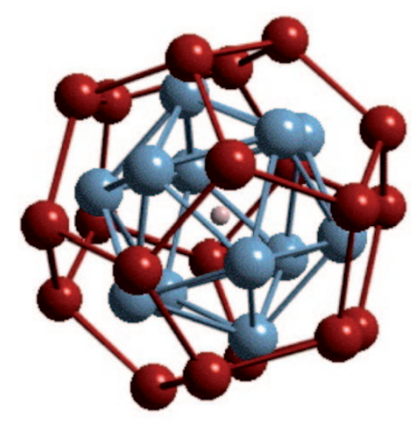

$\mathrm{b}$

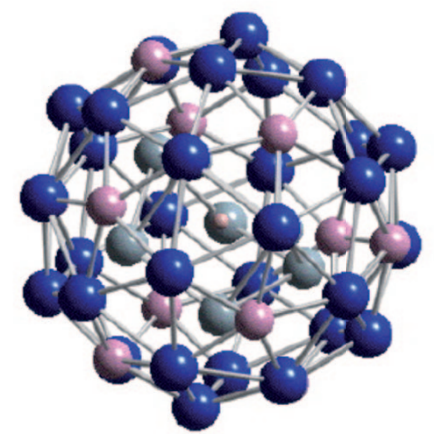

C

3. (a) Exemple typique d'une structure icosaédrique (i-AIPdMn et i-AlCuFe) constituée principalement de l'imbrication de deux types d'amas atomiques, type Bergman (b) avec 33 atomes et type Mackay (c) avec 51 atomes. Les espèces chimiques se répartissent de façon ordonnée sur les orbites (repérées par différentes couleurs) de ces deux types d'amas.

\section{Quelques éléments de quasicristallographie}

Dans le cas le plus simple où $N=2$ et $d=1$, on considère dans le plan un réseau carré simple $\Lambda$. On copie en chaque nœud du réseau un segment de droite prototype $\sigma$. On coupe l'ensemble par une droite quelconque $E_{\|}$de pente irrationnelle par rapport aux directions de base du réseau (voir figure). On obtient ainsi une séquence de segments longs et courts, correspondant aux projections sur cette droite des arêtes selon $x$ ou $y$ des carrés. Un objet périodique du plan a engendré, par coupe d'orientation irrationnelle d'une droite, un objet quasi-périodique à une dimension sur cette droite. Dès que la pente est rationnelle (droite verte de la figure), la séquence est périodique. Les quasicristaux sont aux cristaux ce que les nombres irrationnels sont aux nombres rationnels : ils viennent enrichir la panoplie des solides ordonnés à longue distance et partagent avec les cristaux la totalité de leurs propriétés géométriques, sauf la périodicité.

L'application explicite de la méthode au cas des quasicristaux réels conduit, en lieu et place du plan, droites et segments de droite utilisés pour le cas 1D précédent, à considérer un réseau dans un grand espace de dimension 6, et faire des coupes de dimension 3 selon I'espace physique $E_{\|}$et son orthogonal $E_{\perp}$ de surfaces atomiques de dimension 3 (optimalement des polyèdres). À partir de quoi il devient possible $\mathrm{d}^{\prime}$ indexer les spots de diffraction directement dans le grand espace 6D et d'y effectuer toutes les opérations de Fourier usuelles de la cristallographie... mais à 6 dimensions au lieu de 3 .

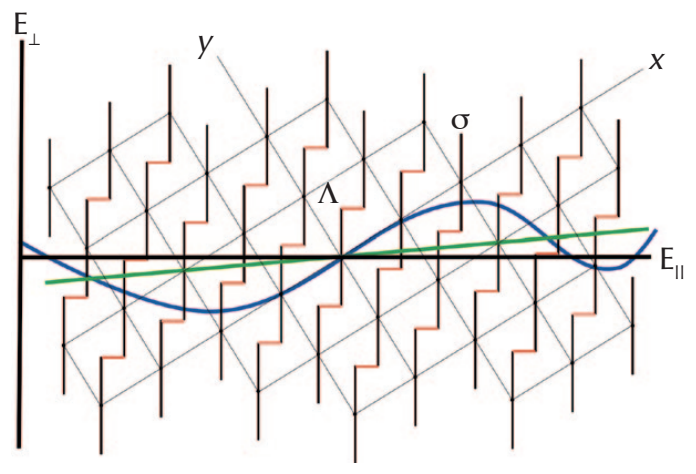

Illustration de l'algorithme de coupe et projection pour générer une structure quasi-périodique à une dimension. (a) Coupe irrationnelle du réseau (droite noire) : modèle du quasicristal parfait de Katz et Duneau.

(b) Coupe rationnelle (droite verte) : structure périodique approximante du quasicristal. (c) Courbe bleue : modèle de Blech, disposition aléatoire d'icosaèdres parallèles connectés par une arête.
Pour les propriétés de transport, ces composés métalliques ont des résistivités à basse température de l'ordre de grandeur de celles des semi-conducteurs dopés et qui varient en loi de Matthiessen` inverse, où ce sont les conductivités des défauts et des phonons qui se somment, au lieu des résistivités. Ce phénomène pourrait être dû à une forte localisation électronique dans les amas atomiques de haute symétrie, rendant ainsi difficiles les sauts électroniques d'un amas à l'autre. Ceci se retrouve dans les propriétés de conduction thermique : les quasicristaux sont de bons isolants thermiques à basse température et pourraient être utilisés, par exemple, comme revêtements thermiques dans les récipients de gaz liquéfiés.

Du point de vue dynamique de réseau, les quasicristaux présentent de nombreux sites atomiques voisins mutuellement exclusifs, où les atomes peuvent sauter d'un site à l'autre. On désigne improprement ces sauts sous le terme "phasons ", par analogie avec les phasons ${ }^{\star}$ des phases incommensurables. Mais contrairement à ces derniers, ceux des quasicristaux sont des sauts à des distances finies, des mouvements discontinus mal décrits par des développements analytiques. 
\>

On s'accorde à penser que ces modes de phasons sont fortement dissipatifs ; l'origine de cette dissipation est encore mal comprise.

Les propriétés mécaniques de ces composés présentent une grande similitude avec celles des intermétalliques complexes à grandes mailles (phases de Laves ou de Frank-Kasper) : un comportement fragile à basse température et ductile au voisinage de la température de fusion. On a identifié les défauts de plasticité en terme de dislocations quasicristallines se mouvant par montée [21]. Leur description géométrique est finalement assez simple comparée aux schémas subtils invoqués pour comprendre la déformation plastique à haute température des intermétalliques complexes. De façon inattendue, les dislocations quasi-cristallines dans ces structures périodiques, appelées alors " métadislocations ", pourraient venir simplifier nos descriptions de la déformation plastique de certains matériaux.

\section{Glossaire}

Icosaèdre

Polyèdre contenant 20 faces l'icosaèdre régulier convexe comporte 30 arêtes, 12 sommets et 6 axes de symétrie d'ordre 5 .

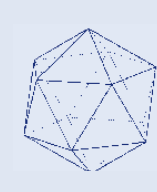

Phases de Hume-Rothery

Alliages formés de métaux de différentes électronégativités, et dont la structure cristalline est déterminée par le nombre moyen d'électrons de valence par atome.

\section{Pseudo-gap}

Minimum profond dans la densité d'états électroniques à l'énergie de Fermi.

\section{Loi de Matthiessen}

Cette règle, qui suppose que les différents mécanismes de diffusion des électrons sont indépendants, est bien vérifiée expérimentalement dans les alliages métalliques dilués ; la résistivité électrique est alors la somme d'une contribution indépendante de la température, due à la diffusion des électrons par les impuretés et les défauts cristallins, et d'une contribution due aux vibrations atomiques et qui augmente avec la température.

\section{Phasons}

Dans une structure modulée incommensurable, qui peut être décrite comme une structure cristalline périodique à laquelle est superposée une modulation périodique de composition ou de position, incommensurable avec le réseau de base, le phason est un mode d'oscillation de la phase de la modulation.

\section{En guise de conclusion}

La nature n'a certainement pas dit son dernier mot sur la façon dont les atomes se répartissent dans les solides : la quasipériodicité n'épuise pas toutes les solutions ordonnées possibles, loin s'en faut ! De nombreux autres algorithmes déterministes de complexité finie existent. Ils donnent naissance à des objets parfaitement ordonnés à longue distance, qui ne sont ni périodiques ni quasi-périodiques. Certains d'entre eux seront sans doute découverts un jour dans la nature et requerront, à leur tour, de nouveaux outils avec de nouvelles symétries cachées. On ne peut que souhaiter que les organismes français de recherche soient alors aussi réactifs que l'ont été, en leur temps, le CNRS, le CEA et le ministère des Universités à l'occasion de la découverte des quasicristaux. En effet, l'Europe y a joué un rôle de pionnier, d'abord en France avec le Groupe de Recherche (CNRS) Quasicristaux jusqu'aux années 90, et à partir de 1997 du Schwerpunkt Quasikristalle (DFG) en Allemagne. Ensemble, ils auront fédéré une bonne centaine de laboratoires européens avec de très confortables moyens financiers.

Plus récemment, dans un esprit plus spécifiquement science des matériaux, a été créé un réseau européen d'excellence sur les composés intermétalliques complexes, concernant les applications possibles de ces étonnantes structures métalliques. On trouvera l'état de l'art actuel de la recherche sur les quasicristaux dans la référence [22].

(1) Rappelons que la symétrie de translation d'un réseau cristallin périodique à 3 dimensions n'est compatible qu'avec des axes de symétrie d'ordre $\mathrm{n}=2$, 3,4 et 6 (correspondant à des rotations d'angle $2 \pi / \mathrm{n}$ transformant le cristal en lui-même).

\section{Les quasicristaux, en bref...}

Un quasicristal est un solide dont la structure n'est pas périodique (contrairement à un cristal classique), mais qui présente un ordre à longue distance se traduisant par un spectre de diffraction dénombrable. La plupart des quasicristaux sont thermodynamiquement instables, mais il en existe qui sont stables.

Par exemple, les quasicristaux icosaédriques (souvent des alliages ternaires) présentent une symétrie d'ordre 5, incompatible avec la symétrie de translation de réseau. Leur structure atomique peut être décrite approximativement comme une imbrication de deux ou plusieurs types d'amas de quelques dizaines d'atomes et de symétrie icosaédrique. Les quasicristaux présentent des propriétés physiques (électriques, thermiques, mécaniques, vibrationnelles) originales.

\section{Références}

$1 \bullet Y$. Meyer, Algebraic numbers and harmonic analysis, North-Holland (1972)

2• R. Penrose, Bull. Inst. Math. Appl. 10 (1974) 266, Mathematical Intelligencer 2 (1979) 32-37; voir aussi M. Gardner, Scientific American 236 (1977) 110-121.

3• A. Mackay, Sov. Phys. Crystallogr. 26 (1981) 517-22 Physica 114A (1982) 609-13.

4• D. Shechtman et I. Blech, Met Trans. 16A (1985) 1005-12.

5• D. Shechtman et al., Phys. Rev. Lett. 53 (1984) 1951-53.

6• D. Levine et P. J. Steinhardt, Phys. Rev. Lett. 53 (1984) 2477-80.

7• M. Duneau et A. Katz, Phys. Rev. Lett. 54 (1985) 2688.

8・ V. Elser, Acta Cryst. A42 (1986) 36-43.

9• P.A. Kalugin et al., J. Physique Lett. 46 (1985) 601-7.

10• P.M. de Wolff, Acta Cryst. A33 (1977) 493.
11• A. Janner et T. Janssen, Phys. Rev. B 15 (1977) 643

12• L. Pauling, Nature 317 (1985) 512-14.

13- "Pauling's model not universally accepted", série de réponses à la lettre de L. Pauling, Nature 319 (1986) 102-104.

14• L. Bendersky, Phys. Rev. Lett. 55 (1985) 1461.

15• B. Dubost et al., Nature 324 (1986) 48-50.

16• A. P. Tsai et al., J. Mater. Sci. Lett. 6 (1987) 1403-5.

17• M. Cornier-Quiquandon et al., Phys. Rev. B 44 (1991) 2071.

18• P. Guyot et M. Audier, Phil. Mag. B 52 (1985) L15-L19.

19• M. Boudard et al., J. Phys. Cond. Matter 4 (1992) 10149.

20 J. Friedel et F. Dénoyer, CRAS Paris 305 (1987) 171.

21• D. Caillard et al., Scripta Mat. 49 (2003) 11.

22- "Proceedings of the Eleventh International Conference on Quasicrystals", Phil. Mag. 91, Special Issue 19-21 (2011).

\section{Ouvrages aénéraux}

- The Physics of Quasicrystals, éditeurs P. J. Steinhardt et S. Ostlund, World Scientific, Singapour (1987).

- C. Janot, Quasicrystals: a primer, Oxford University Press, Oxford (1992).

- M. Duneau et C. Janot, La magie des matériaux, Odile Jacob (1995) 